\title{
APORTES DE ALEXANDER CHAYANOV A LOS ESTUDIOS DE LA ANTROPOLOGÍA ECONÓMICA Y RURAL
}

\author{
Lic. Karina FLEITAS ${ }^{1}$
}

Dra. Magali PAZ ${ }^{2}$

\author{
Dr. Sebastián VALVERDE ${ }^{3}$
}

\section{Resumen}

En este artículo nos proponemos analizar los principales planteos del agrónomo Ruso A. Chayanov (1890-1930) cuyos aportes fueron claves para el análisis de la denominada Economía Campesina o "Unidad Económica Campesina". En primer lugar, retomaremos el contexto de Rusia a fines del Siglo XIX y principios del XX, fundamental para comprender sus planteos y el debate con los autores marxistas. Luego, estudiaremos los principales aspectos por los cuales su obra tuvo una gran repercusión en la Antropología Económica y Rural. Finalmente, analizamos cómo sus categorías fueron retomadas desde la Antropología en América Latina y, en particular, en Argentina en los años '60 y '70.

Palabras clave: Análisis socio-antropológico; Capitalismo agrario; Unidad campesina; Auto explotación; Teoría marxista.

\footnotetext{
${ }^{1}$ Centro de Estudios e Investigaciones sobre Cultura y Sociedad (CIECS)- Universidad Nacional de Córdoba (UNC)

${ }^{2}$ Centro de Estudios e Investigaciones sobre Cultura y Sociedad (CIECS)- Universidad Nacional de Córdoba (UNC) - CONICET

${ }^{3}$ CONICET - Instituto de Ciencias Antropológicas - Facultad de Filosofía y Letras-Universidad de Buenos Aires / Universidad Nacional de Luján(UNLu) sebavalverde@gmail.com
} 


\begin{abstract}
In this article we propose to analyze the main proposals of the Russian agronomist Alaxander Chayanov (1890-1930) whose contributions were key to the analysis of the so-called "Peasant Economy" or "Peasant Economic Unity". In the first place, we will return to the context of Russia at the end of the 19th and beginning of the 20th century, fundamental to understanding its proposals and the debate with Marxist authors. Then, we will study the main aspects by which his work had a great repercussion in Economic and Rural Anthropology. And finally, we analyze how their categories were taken up from anthropology in Latin America and, in particular, in Argentina in the 60s and 70s. Keywords: Economic Anthropology; "Peasant Economy; Peasant unit; Alaxander Chayanov
\end{abstract}

\title{
Résumé :
}

Dans cet article, nous proposons l'analyse des principales propositions de l'agronome russe Alaxander Chayanov (1890-1930) dont les contributions ont été essentielles à l'analyse de la soi-disant «économie paysanne» ou «unité économique paysanne». En premier lieu, nous reviendrons sur le contexte de la Russie à la fin du XIXe siècle et au début du XXe, qui est essentiel pour comprendre ses propositions et le débat avec les auteurs marxistes. Ensuite, nous étudierons les principaux aspects pour lesquels ses travaux ont eu un grand impact sur l'anthropologie économique et rurale. Et, enfin, nous analysons comment leurs catégories ont été reprises de l'anthropologie en Amérique latine et, en particulier, en Argentine dans les années 60 et 70.

Mots clés: Anthropologie Ëconomique; «Économie paysanne; Unité paysanne; Alaxander Chayanov

\section{Introducción ${ }^{4}$}

"Nosotros contraponemos, y lo seguimos haciendo, la explotación doméstica a la capitalista, -y lo hemos hecho al nivel de la organización de la producción-la explotación doméstica se contrapone a la basada en fuerza de trabajo de terceros"

(Chayanov, 1974: 225).

La corriente estructural-funcionalista de nuestra disciplina ha consagrado en los estudios antropológicos el par de opuestos sociedad tradicional-sociedad moderna y, cualquiera sea la valoración (positiva o negativa) que se haga de lo rural, siempre la idea de

\footnotetext{
${ }^{4}$ Agradecemos a Pamela Pulcinella, por la atenta lectura del texto.
} 
desarrollo o progreso es presentada como un proceso en el cual se rompen lazos primordiales, comunitarios, orgánicos y de solidaridad social, mientras se van consolidando relaciones de contrato, impersonales y cuyo ámbito clásico de existencia y reproducción es el mercado. Bajo estas miradas, el polo rural, visto como atrasado o tradicional es concebido como un obstáculo resistente al cambio y poco receptivo a los valores del progreso.

En relación con estas concepciones, nos interesa remarcar, en primera instancia, que la heterogeneidad de las unidades campesinas está fuera de toda duda, pues no pueden ser comprendidas o siquiera descritas de manera apropiada fuera de su escenario societal general. Lo mismo puede decirse del contexto histórico. En segundo lugar, no se puede negar "la dualidad del carácter conservador y progresista de los sujetos sociales que desde las parcelas y las haciendas han actuado tanto resistiendo como motorizando transformaciones económicas y sociales" (Peón 1992: 9). El autor plantea que la tendencia a ignorar la dualidad de los sujetos agrarios ha inducido a juicios de valor erróneos sobre sus potencialidades transformadoras, lo que provoca aseveraciones absolutas que bloquean la posibilidad de investigar cada caso particular y buscar las especificidades a lo largo de la historia.

Si tomamos en cuenta las cuestiones aludidas, se puede observar que la primera va en directa relación con la segunda: es preciso ahondar en una caracterización y análisis de la "heterogeneidad" del mundo campesino o de los pequeños productores para comprender la capacidad de organización, lucha o resistencia que suelen tomar estos grupos sociales ante determinados contextos opresivos, especialmente en un continente como el nuestro, con su historia de colonización y esclavitud en el ámbito rural (desde la mita andina y las haciendas hasta llegar al latifundio y las empresas capitalistas del agro contemporáneo).

En este sentido, reconocemos que los pioneros trabajos de Malinowski (1972), Mauss (1971), Sahlins (1983), Meillassoux (1977), Polanyi (1976), Godelier (1979) y Wolf (1971), entre los más destacados, permitieron enriquecer el sentido de la Antropología Económica, ya que estudiaron diversas formas de hacer y pensar al homo economicus (lejos de la tan mentada "racionalidad económica unificada"), y esclarecieron fenómenos centrales en torno a economías que operaban sobre bases totalmente distintas a las de la economía occidental moderna y cómo comprender aquella variedad de instituciones que no eran el mercado clásico y en las que se hallaba incrustada la subsistencia de muchas comunidades. Estos son los antecedentes que luego se desarrollarían en las décadas de 1960 y 1970 en el debate entre formalistas y sustantivistas dentro de la disciplina ${ }^{5}$. De

\footnotetext{
${ }^{5}$ A grandes rasgos, podemos decir que los formalistas asumen como propia la definición neoclásica de la ciencia económica que estudia al comportamiento humano como una relación entre fines y medios escasos cuyos usos alternativos no se inmutaban ante el riesgo del etnocentrismo. Esto es, la vertiente formalista va a universalizar los supuestos subjetivistas neoclásicos de elección racional y escasez. Los sustantivistas, en cambio, asumen la definición clásica de la ciencia económica y ponen énfasis en la dependencia de las personas respecto a la naturaleza y a otras personas para obtener su sustento. No hay escasez por definición, sino que hay formas diferentes de distribuir los recursos y los bienes producidos en cada cultura. "La institucionalización es el eje clave en el concepto sustantivo de economía. Las formas de organización e
} 
manera general, el eje de controversia fue determinar qué tipo de diferencias existían entre la dinámica productiva "primitiva" (en sus variantes de economías campesinas, de agricultura de roza, cazadoras-recolectoras o pastoriles) y las capitalistas.

Frente a este panorama, los estudios de autores como Sahlins (1983), Godelier (1986, 1987) y el mencionado Meillassoux, más allá de sus diferentes enfoques, concordaron en abordar estos sistemas económicos delimitando sus rasgos y su lógica intrínseca de funcionamiento.

Es aquí donde nuestra mirada necesariamente va dirigida a un autor que ha sido una referencia ineludible en la Antropología Económica y Rural: el agrónomo ruso A. V. Chayanov (1890-1929), que perteneció a la denominada "escuela para el análisis de la organización y producción campesinas". La importancia y repercusión de sus planteos radica, precisamente, en efectuar una serie de formulaciones acerca de la especificidad de la economía campesina y plantea que esta posee una lógica propia diferenciada de la capitalista. Luego retomaremos y profundizaremos este punto.

El contexto en que se enmarca la teoría de Chayanov es el de los años posteriores a la Revolución Rusa - mucho antes del desarrollo de la Antropología Social y la Antropología Económica y Rural como subcampo de la primera-, época en que se discutió acerca de las características de las comunidades rurales del país (Paz 2016). Por ello, sus investigaciones se desarrollaron a la luz de los intensos debates que tuvieron lugar en dicho país sobre la cuestión agraria a fines del siglo XIX y principios del XX.

Como adelantamos, este autor constituye una referencia ineludible en cualquier introducción a la Antropología Económica o Rural, hecho comprensible a partir de una multiplicidad de factores, que precisamente analizaremos en este trabajo.

A. V. Chayanov (1974) [1925] ha caracterizado al campesinado como un tipo específico de economía, cuya actividad está determinada por las necesidades del grupo doméstico las que van cambiando a lo largo del ciclo familiar. En su obra central, La organización de la unidad económica campesina (1974 [1925]), sostuvo que la economía campesina no se basaba en el mismo tipo de cálculo que la empresa capitalista, sino que se orientaba hacia las necesidades de consumo de la unidad doméstica. Este es uno de los puntos centrales de sus planteos y quizás una de las aristas que más contribuyeron en la fuerte repercusión que tuvo su obra.

A partir de estos antecedentes que hemos expuesto, deseamos especificar los objetivos del artículo, que se basarán, precisamente, en analizar los principales planteos del agrónomo Ruso A. Chayanov cuyos aportes fueron claves para el análisis de la denominada Economía Campesina o "Unidad Económica Campesina". Retomaremos el contexto de Rusia a fines del siglo XIX y principios del siglo XX, que resulta fundamental para comprender sus planteos y el debate con los autores marxistas, cuyos lineamientos 
abordaremos brevemente para comprender estos debates. Analizaremos las causas por las que su obra tuvo gran repercusión en la Antropología Económica y Rural y, a la vez, cómo sus categorías luego fueron retomadas en las décadas de 1960 y 1970 desde la Antropología. En la última parte del artículo, abordaremos cómo va a ser releído en América Latina y Argentina, atendiendo también a sus límites y a la búsqueda de adaptar sus categorías a los estudios locales.

En este trabajo se ven plasmados los resultados de años de investigaciones desarrolladas por los autores.

Karina Fleitas es licenciada en Historia (Facultad de Filosofía y Humanidades, Universidad Nacional de Córdoba), y actualmente está finalizando su doctorado en Ciencias Antropológicas (Universidad de Buenos Aires). Sus trabajos de investigación analizan, desde la Antropología Rural y Económica, el proceso de avance de la frontera agropecuaria en zonas extrapampeanas, y pone el foco de atención en los efectos socioeconómicos ocasionados sobre las familias campesinas y pequeños productores rurales por la apropiación, manejo y distribución del agua en el noroeste de la provincia de Córdoba, región centro de la Argentina.

Magalí Paz es doctora en Antropología (Facultad de Filosofía y Letras, Universidad de Buenos Aires) y, desde 2018, es becaria posdoctoral del Consejo Nacional de Investigaciones Científicas y Técnicas (CONICET) cuyo lugar de trabajo es el Centro de Investigaciones y Estudios sobre Cultura y Sociedad (CIECS). Magalí analiza los efectos generados por el avance de la frontera ganadera en el noroeste de la provincia de Córdoba (disputas por el acceso, la disponibilidad, apropiación, distribución y gestión de los recursos naturales y estatales). Específicamente, y desde una perspectiva antropológica, estudia cómo dichos procesos impactan sobre la población rural más vulnerable: los pequeños productores, cosecheros hortofrutícolas y campesinos.

Por último, Sebastián Valverde, antropólogo social de formación de grado y posgrado en la Facultad de Filosofía y Letras de la Universidad de Buenos Aires, investiga desde hace años la conflictividad territorial del pueblo mapuche en relación con los procesos de actualización étnico-identitaria, las transformaciones socioeconómicas regionales y el desarrollo de los movimientos indígenas. Es docente en las asignaturas de Antropología Económica y el seminario curricular de Antropología Rural en la Facultad de Filosofía y Letras (UBA). Ha escrito textos teóricos, junto con otros autores, sobre la Antropología Económica y Rural.

\section{El contexto histórico de la teoría de A. V. Chayanov y los primeros antecedentes teóricos}

Para poder comprender en todas sus dimensiones la obra de A. V. Chayanov, es necesario retrotraernos al "contexto de época" y, en particular, a los debates que tuvieron lugar en el periodo entre 1890 y la Revolución Rusa (1917) y, posteriormente, hasta las medidas de colectivización en la década de 1930. En efecto, toda esta etapa está marcada por la polémica entre populistas y la "escuela para el análisis de la organización y producción 
campesinas" (de la que formaba parte Chayanov) y marxistas (Archetti 1974). Para estos últimos -en el contexto de la Revolución Rusa de 1917 y el proceso de transformación social en su conjunto-, el foco principal del análisis y la preocupación estaban puestos en la manera en que el avance de las relaciones capitalistas de producción establecía diferencias entre los campesinos, lo que obligaba a unos a vender su fuerza de trabajo, mientras otros se capitalizaban y contrataban a otros como asalariados (Balazote y Radovich 1992; Schiavoni 1998). Estas últimas lecturas son conocidas como las de la diferenciación social, cuyos principales exponentes fueron el propio Lenin, líder político de la Revolución Rusa (1870-1924) $)^{6}$ y Kautsky (1854-1938) ${ }^{7}$. En el siguiente apartado profundizaremos en estas categorías.

Chayanov en cambio, centró mayormente el análisis y la preocupación en las diferenciaciones entre las familias y su ciclo de desarrollo, es decir, la denominada diferenciación demográfica.

Por su parte, en su análisis de las clases sociales, Lenin clasifica los campesinos en ricos, medianos y pobres, y utiliza como criterio la compra o venta de la fuerza de trabajo. Los campesinos ricos compran fuerza de trabajo adicional, los medianos usan la propia fuerza de trabajo y los pobres la venden (a otro que tiene la capacidad de comprarla).

Otro aspecto que se debe tener en cuenta para situarnos en el contexto de producción de la obra de Chayanov es la gran cantidad de estadísticas que se venían recabando por aquellos años. Es importante hacer notar que, al organizarse los Zemtsevos después de la reforma, en toda Rusia se recogían sistemáticamente datos sobre el tamaño de las explotaciones, tipo de cultivos, tamaño de la familia y su composición por edad, pautas de consumo, producción y artesanías (Archetti 1974).

El hecho de que en el ámbito del campo el modo de producción predominante fuera el mercantil simple, que la Rusia de ese momento fuera un país con $85 \%$ de población campesina, que la economía dependiera de su sector externo agrícola, que en su seno se desarrollaran diferentes escuelas pero todas estuvieran abocadas al sector agrario y que se dispusiera de un vasto material estadístico, eran condiciones suficientes para la producción de un conocimiento sustantivo de la situación social y económica sobre el comportamiento del campesinado.

En efecto, a partir de 1911 Chayanov va a dedicar toda su obra a la construcción de una teoría diferente de la de economía capitalista, dado además los problemas que se planteaban en relación a la modernización y tecnificación del campo ruso (Archetti, 1974).

\footnotetext{
${ }^{6}$ Lenin, V. I. (1950): El desarrollo del capitalismo en Rusia. El proceso de la formación de un mercado interior para la gran industria - Moscú: Ediciones en Lenguas Extranjeras.

${ }^{7}$ Kautsky, K. (1899): "La cuestión agraria. Estudio de las tendencias de la agricultura moderna y de la política agraria de la socialdemocracia". en La cuestión agraria (Die agrarfrage) - C. Kautsky, C. (trad. Ciro Bayo), Barcelona: Editorial Laia.
} 
El régimen que predominaba en Rusia en aquel entonces era la denominada "comuna" por el cual -tal como señala Archetti:

Cada familia campesina, de acuerdo con el tamaño y la relación existe entre los miembros en condiciones o no de trabajar, recibía de la comuna una determinada cantidad de hectáreas para su uso. La partición de una familia al separarse un hijo para formar su familia no alteraba todo el ciclo, pues aquel se presentaba ante la comuna y solicitaba su pedazo de tierra (Archetti 1974: 18).

En este sentido, A. V. Chayanov (1974) ha caracterizado al campesinado como un tipo específico de economía, cuya actividad está determinada por las necesidades del grupo doméstico. Por ello, los conceptos de "renta" y "salario" no son aplicables en este contexto.

Esto hace que para el autor la economía campesina constituya un modo de producción en el mismo nivel que el esclavista o el capitalista. Esta es una de las diferencias con Marx, para quien la producción mercantil simple nunca llega a constituirse en un modo de producción dominante y, como tal, puede estar presente y desarrollarse bajo diferentes modos de producción (Archetti 1974).

Chayanov ha teorizado acerca de la relación entre el balance y el consumo, que condiciona el esfuerzo productivo de la familia a lo largo de su ciclo de vida. En su análisis se centró en la importancia de satisfacer las necesidades familiares, que van cambiando a lo largo del ciclo familiar.

La pregunta central que intenta responder el autor es la siguiente: ¿qué mecanismos económicos están por detrás de las unidades de producción que utilizan en el proceso productivo la fuerza de trabajo familiar?

Según refieren Archetti y Stølen :

de un lado de la ecuación están el tamaño y la relación existentes, en el seno de la familia, entre los que trabajan y no trabajan. La combinación entre estos recursos y los medios de producción, tierra, herramientas de trabajo, está mediada por la dimensión subjetiva, propia a este tipo de economía: la intensidad del trabajo o, en otras palabras, el grado de auto-explotación de la fuerza de trabajo familiar (1975: 111).

En efecto, para Chayanov - como ya fuera aludido-, el trabajo campesino persigue como fin la satisfacción de sus necesidades y, por ello, el núcleo de sus análisis es el balance entre consumo familiar y explotación de la fuerza de trabajo. Así, afirma: "el principal objeto de las operaciones y transacciones económicas del campesino es la subsistencia y no la obtención de la tasa normal de ganancia” (Chayanov 1974: 221). 
La noción de "autoexplotación" del campesino que forma parte fundamental de esta teoría, sostiene que el trabajo doméstico campesino se desarrolla de acuerdo a una lógica de producción que se traduce en un inmediato consumo (Paz 2016). La forma en que se organiza el trabajo familiar tiene que ver con una dimensión subjetiva en la que intervienen como ecuación, por un lado, el tamaño y las relaciones existentes en el seno de la familia entre los que trabajan y los que no; y por otro lado la combinación entre estos recursos y los medios de producción - tierra, herramientas de trabajo- mediada por la propia intensidad del trabajo. De la interrelación entre variables y decisiones que toma la familia campesina es como se establece el grado de autoexplotación de la fuerza de trabajo familiar (Chayanov 1974: 8).

De esta forma, el análisis de Chayanov intenta explicar, a partir de mecanismos específicos, cómo funciona la economía campesina como un modo de producción concreto, con sus propias leyes. Aunque no profundizaremos en las diatribas en relación al concepto de "modos de producción", sí insistiremos sobre algunos puntos que sobresalen en el análisis y que generan inquietantes preguntas y líneas para el debate actual en torno a la problemática agraria.

A raíz de todo lo expuesto, podemos efectuar esta pregunta central: ¿cómo define exactamente el concepto de economía campesina el autor? En pocas palabras, a partir de dos criterios básicos: 1. Predominio de la fuerza de trabajo doméstica; y 2. Ausencia de una acumulación sistemática de capital. Un campesino tiende a maximizar la autonomía de la explotación en términos del uso de la fuerza de trabajo de terceros y, a la vez, no acumula capital por las razones apuntadas anteriormente. Por el contrario, la organización capitalista de la empresa rural parte de otros supuestos: utilización de fuerza de trabajo asalariada en forma permanente y acumulación de capital.

Otro aspecto clave que debemos pensar en relación con la problemática de universalidad/particularidad de las teorías remite a la aplicabilidad de las formulaciones de Chayanov, quien no pensaba que su teoría fuera universalmente aplicable (Archetti 1974). De hecho, uno de los aspectos destacados de la introducción de la obra de Chayanov que efectúa Archetti es el siguiente: "[...] no es un formalista preocupado por encontrar las leyes de una ciencia económica general sino básicamente un sustantivista" 8 (1974: 9-10).

Por último, otro de los aportes fundamentales es la diferenciación que el autor establece entre "familia" y "unidad doméstica" o "grupo doméstico". En relación con este punto, señala que "[...] es indudable que el concepto de familia, particularmente en la vida campesina, pocas veces coincide con el concepto biológico que lo subyace y en su contenido intervienen una serie de complicaciones económicas y domésticas" (Chayanov 1974: 48). En línea con esta argumentación: [...] los estadísticos del zemstvo ruso, por ejemplo, al realizar censos en los hogares establecieron que para el campesino el concepto

\footnotetext{
${ }^{8}$ Ver nota al pie N. ${ }^{\circ} 2$ donde señalamos los principales aspectos del debate entre formalistas y sustantivistas.
} 
de familia incluye a las personas que comen siempre en la misma mesa o que han comido de la misma olla" (1974: 48).

Nuevamente, deseamos enfatizar lo meritorio de estos planteos efectuados 20-30 años antes de que la Antropología Social británica los retomara al diferenciar familia de grupo doméstico, conceptualizaciones que retomarán en la Argentina en la década de 1970 autores como Archetti y Stølen (1975).

\section{La teoría marxista de diferenciación social y la teoría de la diferenciación demográfica de Chayanov}

Para poder comprender los planteos de Chayanov y su contraposición con los marxistas, es necesario profundizar en torno a las ideas de los principales autores y los aspectos de este debate.

Para comenzar, es preciso aludir que Marx analizó y profundizó el estudio del agro y su relación con la sociedad. El autor plantea que existe una relación asimétrica entre desarrollo agrario y su funcionamiento dentro del sistema capitalista; proceso en el que se desarrollan y afianzan las contradicciones en el seno de las sociedades. La importancia del análisis marxista en los estudios actuales sobre el agro es comprender cómo se fue produciendo un retroceso en el papel principal de la agricultura a medida que el capitalismo se fue consolidando. Sin embargo, más que un retroceso, nos encontramos con una reconfiguración del papel de la agricultura en las relaciones sociales de producción.

Para Marx la economía campesina es mercantil simple: el campesino está continuamente involucrado en operaciones de compra y venta en el mercado, pero claro está, la circulación simple de mercancías (mercancía-dinero-mercancía) tiene como fin la satisfacción de necesidades y lo que se produce es un intercambio entre equivalentes. En esta misma línea, Chayanov afirma que, en la explotación familiar, la familia equipada con medios de producción emplea su capacidad de trabajo en cultivar la tierra y recibe como resultado cierta cantidad de bienes. Si profundizamos sobre la estructura interna de la unidad de trabajo familiar, no se puede imponer el beneficio neto, la renta y el interés de capital sin la categoría salarios:

El campesino o artesano que produce sin pagar mano de obra recibe, al cabo de un año, determinada cantidad de mercancía que, después de intercambiada en el mercado, forma el producto bruto de su unidad económica. De este producto bruto hay que deducir una cantidad para el gasto material durante el año. Nos queda, entonces, el incremento del valor de los bienes materiales de la familia en un año, es decir, el producto de su trabajo. Este producto de trabajo familiar es la única categoría de ingreso para una unidad de trabajo familiar campesino o artesano porque no hay forma de descomponerlo, aquí no se puede aplicar el cálculo 
capitalista de la ganancia frente a la ausencia de salario y del beneficio neto (Chayanov 1974: 8).

Luego de analizar estos postulados, Archetti y Stølen sostienen:

para Marx el límite es el salario, para Chayanov es la súperexplotación de la fuerza de trabajo; para Marx el campesino abandona el proceso productivo si no se paga al menos su trabajo necesario; para Chayanov esto no ocurre necesariamente ya que una de las estrategias a seguir es la de aumentar la intensidad del trabajo (1975: 115).

Chayanov diferencia lo siguiente: el excedente obtenido es resultado de la mejor ubicación o disponibilidad de los medios de producción, no es por la renta o interés de capital. En sus palabras:

La cuantía del producto del trabajo (excedente) la determinan el tamaño y la composición familiar, el número de miembros capaces de trabajar, la productividad de la unidad de trabajo y el grado de esfuerzo de los trabajadores, el grado de auto explotación mediante el cual, los miembros hacen cierta cantidad de unidades de trabajo al año (Chayanov 1975: 10).

Desde los análisis efectuados desde el marxismo de las sociedades precapitalistas, la pequeña propiedad parcelaria tendería a desaparecer, ya que se encontraría excluida del desarrollo de las fuerzas productivas, formas sociales de trabajo, concentración social de capital, etc. No obstante, las investigaciones de Kautsky (1980), se profundiza en otros aspectos. Dicho autor alega que los estudios de Marx no se concentran, justamente, en la persistencia de las formas precapitalistas en un contexto capitalista, dado que la superioridad de la gran explotación agrícola sobre la pequeña propiedad es indiscutible. Así, la propiedad privada de los medios de producción se opone a la pequeña explotación que, al convivir con la economía capitalista, garantiza su supervivencia en base a la autoexplotación (situación que la expone a un equilibrio muy precario dentro de la estructura agraria).

Por su parte, el aporte del líder de la revolución bolchevique de 1917, Vladimir Lenin (1973) profundiza estas líneas de análisis acerca del irrefrenable proceso de avance de la gran propiedad capitalista, pero le otorga un papel fundamental al campesinado como actor social en un proceso social potencialmente revolucionario. A pesar de que las formas (instituciones) campesinas "traban" el desarrollo capitalista, este se introduce, genera diferenciación social en la comunidad y crea nuevos tipos de población rural (burguesía rural, proletariado del campo), frente al campesinado tradicional que se encuentra desplazado. Asimismo, Lenin caracteriza a la burguesía rural como de propiedad independiente que practica la agricultura comercial (por ejemplo, los farmers), y sostiene que las tierras en arriendo y la contratación de braceros y jornaleros 
caracterizan su modo de producir, y el dinero obtenido por acumulación lo invierten en tierras u operaciones usurarias y comerciales.

Chayanov (1975), por el contrario, se diferencia de Lenin y profundiza el análisis de la organización y producción campesina. La estudia desde la lógica interna de la familia. De acuerdo con este autor, y dado que el campesino es un productor mercantil simple, su actividad económica está basada según las necesidades del grupo doméstico, en la relación entre consumo familiar y explotación de la fuerza de trabajo, es decir que el campesinado trabaja hasta cubrir sus necesidades. La gran diferencia entre producción y organización de consumo familiar y la lógica capitalista consiste, de acuerdo al autor, en que la primera produce para la propia reproducción de la vida y no para la reproducción del capital. Para Chayanov, las explotaciones familiares conservan sus particularidades, pero responden a un sistema de relaciones sociales de producción más estructural insertas en un sistema económico hegemónico.

\section{El interés por "lo campesino" y el "sector doméstico". La "economía doméstica" en las décadas de 1960 y 1970}

Las décadas de 1960 y 1970 estuvieron marcadas por un interés por lo campesino, influenciados por los procesos que se venían registrando de las luchas de los sectores rurales, una revisión de los preceptos del marxismo tradicional y una reivindicación (en algunos casos, diríamos, un "enamoramiento") en especial de las nuevas generaciones de estudiantes y profesionales con experiencias como las de Cuba, Vietnam y China, entre otras.

Anteriormente, la Antropología Social británica ya había retomado diversas cuestiones presentes en el análisis de Chayanov. Dentro de esta preocupación por el ciclo de desarrollo del grupo doméstico, Fortes (en Balazote y Radovich 1992) diferenció tres fases: expansión, fisión y reemplazo.

La primera comienza con el matrimonio y finaliza cuando declina el ciclo de fertilidad femenino. La fisión es la que engloba a los matrimonios de los hijos culminando al casarse el último. Finalmente, la etapa de reemplazo, concluye con la muerte de ambos progenitores o cuando estos abandonan la actividad productiva (Balazote y Radovich 1992: 34).

El contexto de la década de 1960 influyó en el interés por la obra de Chayanov cinco décadas después de ser publicada y traducida del ruso al inglés y, luego, a otros idiomas?.

En estas tendencias, es necesario destacar los aportes de Marshall Sahlins (1983)[1972], en la década de 1970, a partir de una lectura culturalista y particularista. Este autor norteamericano permitió situar las unidades domésticas en un contexto más amplio al

\footnotetext{
${ }^{9}$ La obra principal de Chayanov que ya mencionamos, La organización de la unidad económica campesina, fue publicada en Moscú en 1925. Si bien recién fue traducida al inglés en 1966 (Schiavoni 1998), en América Latina y la Argentina se descubrió su obra en la década de 1970.
} 
plantear la existencia - por medio de las funciones propias de la política- de las relaciones de reciprocidad y redistribución. En efecto, el abordaje se centró en problematizar las dinámicas resultantes de diferentes unidades domésticas articuladas entre sí - lo que define como la denominada "comunidad doméstica" en la que el rol de las relaciones políticas a través del parentesco en particular de los jefes resulta fundamental一.

La perspectiva de Sahlins posibilitó, por un lado, redescubrir a Chayanov y favorecer una lectura más antropológica de este último autor; y, a la vez, facilitó la reflexión acerca de las diversas interrelaciones en el seno de un contexto más amplio que el de las unidades domésticas individuales. La definición de Sahlins (1983) de "comunidad doméstica" constituye, precisamente, una entidad más abarcativa (que incluye varias unidades domésticas interrelacionadas por vínculos políticos, factor que Chayanov no incluyó en su análisis).

Sin desmerecer estos aportes, debemos señalar las agudas críticas que recibió Sahlins (y que quienes escribimos este texto hacemos propias) al no visualizar claramente el marco regional, la dominación colonial y, hasta qué punto, parte de la "modalidad doméstica" que él caracteriza ya estaba siendo transformada por la expansión capitalista en la época en que desarrolla sus teorizaciones o retoma a otros autores.

Por otro lado, debemos mencionar una serie de cambios para comprender las dinámicas ligadas al proceso de descolonización, en especial en Asia y África en la segunda mitad del siglo XX, una vez consolidada la división del mundo imperialista, sobre todo en la década de 1970. Esto conlleva que la lógica de las producciones domésticas comenzara a ser visualizada en relación con el proceso global de la economía capitalista (Paz 2016).

Así es como se fueron desarrollando diversos estudios encuadrados en las lecturas de Marx -efectuadas, principalmente, en Francia- que dieron cuenta de las "economías domésticas" en un contexto más amplio. Estos autores son Godelier (1976) y Meillassoux (1977), en especial en la obra Mujeres, graneros y capitales.

En América Latina, los trabajos de Julian Steward ${ }^{10}$ ([1955] 1993) — primero- y, luego, los de uno de sus discípulos, Wolf $(1971)^{11}$, desplazaron el interés por los contenidos culturales hacia el abordaje de las relaciones estructurales que ligan a los campesinos con el sistema social englobante (Schiavoni 1998). El surgimiento de este enfoque (dentro del cual también se destacaron, posteriormente, Roseberry 1989, Mintz 1996, etc.) se vinculó con una tendencia dentro de la antropología que buscaba, precisamente, estudiar a los pueblos indígenas y campesinos no como comunidades aisladas, sino como un todo (Comas D’Argemir 1998, Balazote 2007) y, de esta forma, romper con los denominados

\footnotetext{
10 Julian Steward fue un antropólogo norteamericano que desarrolló una serie de investigaciones en las décadas de 1940 y 1950, y adscribió a la escuela denominada "neo evolucionista".

${ }^{11}$ Para un análisis detallado de esta escuela, ver el artículo de A. Balazote: "Consideraciones en torno a la Economía Política” (2007). También ver el capítulo de D. Comas D’ Argemir, (1998) “Economía Política y Antropología Económica", que forma parte de la obra Antropología Económica.
} 
estudios de "comunidad" que habían predominado en la década del 1950 a partir de la estudios como los de de Redfield ${ }^{12}$.

Los alumnos de Steward, que analizaron los grupos denominados "folk" que eran abordados hasta ese momento como supuestas "comunidades cerradas", remarcaron la necesidad de analizar los vínculos entre las comunidades campesinas en relación con el contexto más amplio y los vínculos que se establecen con diferentes "agentes foráneos", el marco regional y estas interrelaciones con diversos agentes Una obra de referencia al respecto es Peasants (1966) de E. Wolf (traducida al castellano bajo el título Campesinos en 1971).

A partir de las tendencias que se desarrollaron estos años, se profundizaron los trabajos que revisaban los diferentes "tipos" de campesinos, en función de sus formas diferenciales de inserción en la sociedad mayor (Schiavoni 1998).

En la Argentina, se realizó una destacada producción que recuperó esos planteos, analizó el campesinado y retomó las formulaciones que vinculan a estos sectores sociales con la sociedad global. Estos tempranos aportes fueron los primeros estudios locales efectuados desde la perspectiva de la Antropología Social y la Antropología Rural, no ya desde la tradición folklórica, sino como subdisciplina de la primera. Esto ocurrió hacia la década de 1970, a medida que se fue consolidando la disciplina antropológica y, en particular, esta línea en nuestro país, entre las primeras generaciones de egresados de la carrera Antropología (creada en 1958) ${ }^{13}$.

Estas miradas más totalizadoras favorecieron en las antropólogas y los antropólogos que se dedicaban al campesinado (o a los pequeños productores rurales) un abordaje mucho más abarcador de las dinámicas que los afectaban y configuraban como tales. En el interregno democrático de 1973-1974 se desarrollaron destacadas investigaciones desde

12 Robert Redfield fue un investigador norteamericano que estudió Derecho y luego Antropología. Desarrolló sus investigaciones entre las décadas de 1940 y 1950 en el centro-sur de México. Tuvo una gran impronta en la antropología de aquellos años a partir de conceptos como el continuum "folk-urbano" y el de la "pequeña comunidad", o bien al abordar las relaciones entre lo rural y lo urbano (Contreras y Tonatiuh, 1999).

${ }^{13}$ A partir de 1962, los primeros graduados de la novel carrera de Antropología comenzaron a plantear una ruptura con las concepciones elitistas y asumieron un compromiso con la realidad nacional y con las capas sumergidas de la sociedad. Así surgió una nueva generación que empezó a adherir a lo que se denomina Antropología Social. Poco tiene que ver esta denominación en el ámbito local, con esta misma orientación surgida en Gran Bretaña, enfrentada al culturalismo norteamericano que privilegiaba el estudio de las estructuras sociales de los llamados "pueblos primitivos". En el medio local, en cambio, se la asume como opuesta a la corriente historicista que la enfrentaba taxativamente, al tiempo que se la concebía "como una antropología total, superadora del estudio limitado a los objetos "clásicos"” (Ratier 2010: 32). Sus adherentes no pertenecían a una única línea sino, por el contrario, a varias: estructural funcionalismo, estructuralismo y neomarxismo (Ratier 2010: 32). Muchos de estos nuevos investigadores debieron formarse en las nuevas perspectivas por fuera de la Facultad de Filosofía y Letras de la UBA. Se dictaban cátedras en otras carreras, dado que estos espacios en la antropología estaban vedados a la reflexión crítica, el desempeño laboral y un compromiso con la realidad actual del "otro". En relación con la emergencia y el desarrollo de una serie de investigaciones encuadradas en la antropología social, se pueden consultar los trabajos de Ratier (2010) y Herrán (1990). En relación con la antropología económica, ver Balazote (2007). 
estas perspectivas, cuando estos profesionales -exponentes de las nuevas generaciones- volvieron, con la reinstauración democrática, a ocupar ámbitos universitarios. Entre ellos, podemos mencionar a Vessuri (1976) y Bilbao (1964) para la zona de Santiago del Estero y Tucumán; Bartolomé (1975) para Misiones; Hermitte y Herrán (1977) para Catamarca y Archetti y Stølen (1975) para el norte de Santa Fe.

En este contexto, la lectura de Chayanov fue fundamental. Esto explica la edición traducida al castellano en Argentina en 1974, cuya presentación fue escrita por un autor paradigmático de aquella joven generación de primeros egresados de la novel carrera de Antropología: Eduardo Archetti (1974).

Con el incremento de la represión política hacia fines de 1974 y durante 1975, estas experiencias tuvieron que llamarse a silencio y esta generación debió partir al exilio (Herrán 1990). A partir de la intervención de la Facultad de Filosofía y Letras de la UBA a fines de 1974, predominó la denominada "escuela fenomenológica de la antropología argentina" - hegemonizada por Marcelo Bórmida hasta su muerte en 1979—, quien privilegió el estudio de aspectos descontextualizados de la realidad y efectuó una lectura muy particular de la "fenomenología", que negaba toda teoría y buscaba recuperar la "conciencia mítica" de los indígenas, como forma de llegar a las "esencias culturales". Esta línea teórica continuó hasta el retorno de la democracia en 1983, mientras la Antropología Social permanecía vedada dentro de las universidades y de los organismos de ciencia y técnica. Durante aquellos años estuvo totalmente ausente el análisis de lo "rural" y el carácter mayoritario de pequeños productores de los pueblos indígenas y todo abordaje que los vinculara con dinámicas regionales y globales. La mayoría de las y los autores que debieron partir al exilio de aquella generación de primeros egresados de la carrera de Antropología abandonaron las problemáticas rurales y comenzaron a dedicarse a otros temas.

Luego, con el retorno de la democracia en 1983 comenzaron a consolidarse los diferentes equipos y desarrollaron nuevas producciones que retoman y problematizan en torno a la articulación entre el sector doméstico o la economía doméstica y el contexto más amplio $^{14}$.

\section{Palabras finales}

Las dos posturas que se debatieron intensamente en Rusia durante los primeros años de la Revolución -el enfoque de la diferenciación social de los autores marxistas y el de la diferenciación demográfica de la teoría chayanoviana- resultan de gran trascendencia porque revelan un eje central de esta última que nos interesa enfatizar: el grado de autoexplotación del trabajo campesino. En base a estudios sobre explotaciones campesinas rusas y de otros países, Chayanov pudo establecer que el grado de explotación lo determina un equilibrio particular entre "satisfacción de la demanda familiar y fatiga del trabajo". Esto significa que "cada nuevo rublo obtenido del producto del trabajo

\footnotetext{
${ }^{14}$ Para profundizar en torno a la producción de estos años, ver Trinchero et al. (2007), Trinchero et al. (en prensa 2020) y Balazote (2007).
} 
familiar puede verse de dos formas: desde la importancia para el consumo y saciar la necesidad familiar; en segundo lugar, desde el cansancio, la fatiga con que se produjo" (Chayanov 1975: 10). A su vez, el autor pone evidencia que, con el incremento del producto por trabajo arduo, la valoración subjetiva que tiene para el consumo cada incremento, decrece; y la fatiga de trabajar para ganarlo, que requerirá una mayor autoexplotación, aumenta:

Mientras no se logre el equilibrio entre ambos, la familia que trabaja sin pagar mano de obra continúa con su actividad. Si se llega al punto de equilibrio, no tendría caso seguir trabajando, ya que la energía de trabajo ulterior es más difícil aguantar para el campesino o artesano de lo que significaría renunciar a sus efectos económicos. Por otro lado, la importancia de cada ingreso bruto para el consumo aumenta en una economía familiar con miembros incapaces de trabajar. Así aumenta la auto explotación de la mano de obra familiar, de modo que el nivel de vida de la familia - amenazado por la demanda - se mantenga en cierto modo, igual. (Chayanov 1975: 11).

Por estas razones, y tal como afirma Schiavoni (1995), la importancia del análisis de Chayanov es que "incluye a economías alternativas basadas en el trabajo familiar, el cálculo no monetarizado de las elecciones y la consideración de que existen diferentes tamaños óptimos de empresas y escalas de producción según las actividades y contextos locales". En el pensamiento económico, para este autor, es necesario avanzar en forma completamente diferente a la lógica capitalista, es decir, a la unidad económica familiar no asalariada (aún predominante en la producción y trabajo agrario). De esta forma, a pesar de ser entendida como Unidad Económica Familiar- Unidad Económica de Trabajo - Unidad Económica de Trabajo Familiar y Unidad Económica Familiar de Trabajo, tiene el mismo significado, esto es: "la explotación económica de una familia campesina o artesana que no utiliza obreros pagos sino que utiliza a los mismos miembros de la familia" (Chayanov 1975: 5)

En el balance aquí efectuado es necesario señalar que las tesis de Chayanov soslayaron la importancia de factores que explican el surgimiento de la economía campesina, su permanencia y recomposición, más allá de los mecanismos demográficos. En este sentido, podemos aseverar que su análisis tuvo un sesgo sincrónico o "ahistórico". Su preocupación fundamental fue analizar la unidad familiar desde la perspectiva micro y sus diferenciaciones en función del ciclo de desarrollo; de ahí que a esta teoría se la conozca como de la diferenciación demográfica. Este aspecto, resulta paradójico: precisamente, la mirada micro de Chayanov también es uno de los aspectos clave para comprender el impacto de su obra en la Antropología.

En efecto, en un intento por esbozar las causas que explican la repercusión que ha tenido la obra de este autor ruso, podemos mencionar su interés en la Antropología a pesar de que su profesión era agrónomo. Su análisis fue asimilable al antropológico, precisamente, porque se centra en la familia campesina como unidad de análisis y hace foco en su 
dinámica interna. Como ha señalado Schiavoni, en este punto: "La innovación de Chayanov consiste en comenzar la consideración de la agricultura campesina 'desde abajo', es decir desde la lógica operativa de la familia" (1998: 35). En realidad, no consideró a la familia como tal — desde el punto de vista del parentesco-biológico y reproductivo- sino económico.

La segunda razón para explicar el impacto de su obra es que sus teorizaciones fueron desarrolladas cuatro décadas antes del surgimiento de la Antropología Económica como subcampo dentro de la Antropología ${ }^{15}$. Muchos de los tempranos aportes de Chayanov fueron retomados luego por la Antropología. Tal es el caso de las fases del ciclo de desarrollo doméstico, la diferenciación entre familia y grupo doméstico, temáticas abordadas por la Antropología Social británica o bien las propias lecturas de Chayanov que fueron abordadas años después, como la que efectuó Sahlins en la década de 1960 (señalada anteriormente).

Podemos esbozar una tercera causa y es el hecho que Chayanov haya vivido y desarrollado sus debates en uno de los países del llamado "Socialismo Real" y en pleno proceso revolucionario. Entre las décadas de 1960 y 1970, la mirada estuvo puesta en estos países como parte de los debates que se fueron dando en la academia, en la militancia política-universitaria y, en particular, en las nuevas generaciones, en relación con el rol del campesinado, la revisión y reformulación de los preceptos del marxismo y en las experiencias del socialismo real como la de Rusia - luego conformada junto con otras naciones como URSS-, China y las que estaban comenzando en Cuba, Vietnam y algunos países africanos y asiáticos, etc. En este contexto, podemos decir que la lectura de este autor - que permaneció en el olvido hasta la década de 1960- le agregó una dosis de atractivo, máxime atendiendo a sus debates (y algunos puntos convergentes) con Marx, Lenin, Kautsky, etc.

Para finalizar, cabe destacar que aun con las limitaciones de su abordaje, como es el sesgo ahistorico, el foco en la dinámica interna de la familia y no en el contexto más amplio ni en la interrelación entre las diferentes unidades familiares entre sí (como luego van a abordar diferentes posturas y abordajes a partir década de 1960), el legado de Chayanov a la Antropología Económica y Rural. La trascendencia que ha tenido posibilitó generar diferentes debates y ser un antecedente ineludible en la temática en los años de la conformación disciplinar.

Recuperamos, como forma de cerrar esta contribución con las palabras de Archetti en la presentación a su obra: "Cuando uno vuelve a la polémica entre populistas y marxistas o cuando uno lee a Chayanov, tiene la impresión de que mucha de la literatura posterior

\footnotetext{
${ }^{15}$ Partimos de considerar como momento de surgimiento de la Antropología Económica la década de 1950 cuando se desarrollaron estudios en esta subdisciplina y la obra de Melville Herskovits, antropólogo norteamericano se reeditó (en 1952) bajo la denominación "Antropología Económica" (Trinchero 1998). Una de sus obras más conocidas se titula La vida económica de los pueblos primitivos, cuya primera edición data de 1940. En la década de 1960, se produce el debate formalistas y sustantivistas que se considera fundacional de la disciplina (Trinchero y Balazote 2007, Herskovits 1952).
} 
sobre campesinos y especialmente la antropología, es pura repetición de algo dicho con más pasión" (1974: 9).

\section{Bibliografía}

Archetti, E. (1974): "Presentación" en La organización de la unidad económica campesina - (pp. 7-21) Chayanov, A. V. Buenos Aires: Nueva Visión.

Archetti, E. y Stølen, K. A. (1975): Explotación familiar y acumulación de capital en el campo argentino - Buenos Aires: Siglo XXI.

Bilbao, S. (1964). "Poblamiento y actividad humana en el extremo norte del Chaco santiagueño" - Cuadernos del Instituto Nacional de Antropología y Pensamiento Latinoamericano, 5, 143-162.

Balazote, A. (2007): Antropología Económica y Economía Política - Córdoba, Argentina: Centro de Estudios Avanzados, UNC.

Balazote, A. y Radovich, J. C. (1992): “El concepto de grupo doméstico” en Antropología Económica II (pp. 27-43) - Trinchero, H. (Comp.) Buenos Aires: Centro Editor de América Latina.

Bartolomé, L. (1975): “Colonos, plantadores y agroindustrias. La explotación agrícola familiar en el sudeste de Misiones"- Desarrollo Económico, 15, 239-264.

Chayanov, A. V. (1974): La organización de la unidad económica campesina - Buenos Aires: Nueva Visión.

Comas D’ Argemir, D. (1998): Antropología Económica - Barcelona: Ariel.

Contreras, R. y Tonatiuh, A. (1999). "Robert Redfield y su influencia en la formación de científicos mexicanos" - Ciencia Ergo Sum, 2(6) México: Universidad Autónoma del Estado de México Toluca 
Papeles de Trabajo No 40 - Diciembre 2020 - ISSN 1852-4508

Centro de Estudios Interdisciplinarios en Etnolingüística y Antropología Socio-Cultural

Dufy, C. y Weber, F. (2007): L'ethnographie économique - París: La Découverte, Repères.

Godelier, M. (1976): "Es posible una antropología económica" en Antropología y Economía - Godelier, M. (Comp.) Barcelona: Anagrama.

Godelier, M. (1986): La producción de grandes hombres. Poder y dominación masculina entre los Baruya de Nueva Guinea - Madrid: Akal.

Hermitte, E. y Herrán, C. (1977): "Sistema productivo, instituciones intersticiales y formas de articulación social en una comunidad del noroeste argentino" en Procesos de articulación social - E. Hermitte y L. Bartolomé (Eds.) (pp. 238-256) Buenos Aires: Amorrortu Editores.

Herrán, C. (1990). "Antropología Social en la Argentina: Apuntes y perspectivas" Cuadernos de Antropología Social, 10, 108-114.

Herskovits, M. [1940] (1952): Antropología económica. Estudio de Antropología Comparada - Ciudad de México: Fondo de Cultura Económica.

Kautsky, K. (1899): "La cuestión agraria. Estudio de las tendencias de la agricultura moderna y de la política agraria de la socialdemocracia" en La cuestión agraria (Die agrarfrage) - Kautsky, C. (trad. Ciro Bayo) Barcelona: Editorial Laia.

Lenin, V. I. (1950): El desarrollo del capitalismo en Rusia. El proceso de la formación de un mercado interior para la gran industria - Moscú: Ediciones en Lenguas Extranjeras.

Malinowski, B. (1972): Los Argonautas del pacífico occidental - España: Península.

Mauss, M. (1971): Sociología y antropología. Segunda Parte. Ensayo sobre los dones. Motivo y forma del cambio en las sociedades primitivas - Madrid: Tecnos.

Meillassoux, C. (1977): Mujeres, graneros y capitales - México: Siglo XXI. 
Papeles de Trabajo No 40 - Diciembre 2020 - ISSN 1852-4508

Centro de Estudios Interdisciplinarios en Etnolingüística y Antropología Socio-Cultural

Mintz, S. (1996): Dulzura y poder. El lugar del azúcar en la historia moderna-México: Siglo XXI.

Paz, M. (2016): Producción, reproducción social y conflictividad por el acceso a los recursos en Unidades Domésticas del departamento Cruz del Eje, Noroeste de Córdoba. Tesis de doctorado - Buenos Aires: Facultad de Filosofía y Letras, Universidad de Buenos Aires.

Peón, C. (1992): Sociología rural latinoamericana - Buenos Aires: Centro Editor de América Latina.

Polanyi, K. (1976): "La economía como proceso institucionalizado" en Antropología y Economía - Godelier, M. (Comp.) (pp. 155-178) Barcelona: Anagrama.

Ratier, H. (2010). "La antropología social argentina: su desarrollo" - Antropología y Ciencias Sociales, 9, 17-47.

Roseberry, W. (1989): "Los campesinos y el mundo" en Antropología económica Plattner, S. (Ed.) (pp. 154-176) Ciudad de México: Consejo Nacional para la Cultura y las Arte (CONACULTA).

Sahlins, M. ([1972]1983): Economía de la Edad de Piedra. Capítulo II (pp. 55-115) Barcelona: AKAL.

Schiavoni, G. (1998): Colonos y Ocupantes. Parentesco, reciprocidad y diferenciación social en la frontera agraria de Misiones - Posadas: Editorial Universitaria, Universidad Nacional de Misiones.

Steward, J. ([1955] 1993): "El concepto y el método de la ecología cultural" en Antropología: lecturas -Bohannan, P. y Glazer, M. (Eds.) (pp. 334-344) Madrid: McGrawn-Hill

Trinchero, H. (1998): Antropología Económica. Ficciones y producciones del hombre económico - Buenos Aires: EUDEBA. 
Trinchero, H. y Balazote, A. (2007): De la Economía Política a la Antropología Económica - Buenos Aires: EUDEBA.

Vessuri, H. (1976). "La explotación agrícola familiar en el contexto de un sistema de plantación: Un caso de la provincia de Tucumán" - Desarrollo Económico, 15(58), 215238.

Wolf, E. (1971): Los Campesinos - Barcelona: Labor. 